Research in Astron. Astrophys. Vol.0 (200x) No.0, 000-000

http://www.raa-journal.org http://www.iop.org/journals/raa

Research in

Astronomy and

Astrophysics

\title{
Grids of rotating stellar models with masses between 1.0 and 3.0 $\mathrm{M}_{\odot}$
}

\author{
Wu-Ming Yang ${ }^{1,2}$, Shao-Lan $\mathrm{Bi}^{1}$ and Xiang-Cun Meng ${ }^{2}$ \\ 1 Department of Astronomy, Beijing Normal University, Beijing 100875, China; \\ yangwuming@ynao.ac.cn; yangwuming@bnu.edu.cn \\ 2 School of Physics and Chemistry, Henan Polytechnic University, Jiaozuo 454000, Henan, China.
}

\begin{abstract}
We calculated a grid of evolutionary tracks of rotating models with masses between 1.0 and $3.0 \mathrm{M}_{\odot}$ and a resolution $\delta M \leq 0.02 \mathrm{M}_{\odot}$, which can be used to study the effects of rotation on stellar evolutions and on the characteristics of star clusters. The value of $\sim 2.05 \mathrm{M}_{\odot}$ is a critical mass for the effects of rotation on stellar structure and evolution. For stars with $M>2.05 \mathrm{M}_{\odot}$, rotation leads to an increase in the convective core and prolongs the lifetime of main sequence (MS); rotating models evolve slower than non-rotating ones; the effects of rotation on the evolution of these stars are similar to those of convective core overshooting. However for stars with $1.1<\mathrm{M} / \mathrm{M}_{\odot}<2.05$, rotation results in a decrease in the convective core and shortens the lifetime of MS; rotating models evolve faster than non-rotating ones. When the mass is located in the range of $\sim 1.7-2.0 \mathrm{M}_{\odot}$, the mixing caused by rotationally induced instabilities is not efficient; the hydrostatic effects dominate the effect on the evolution of these stars. For the models with masses between about 1.6 and $2.0 \mathrm{M}_{\odot}$, rotating models always exhibit lower effective temperatures than non-rotating ones at the same age during the MS stage. For a given age, the lower the mass, the smaller the change in the effective temperature. Thus rotations could lead to a color spread near the MS turnoff in the color-magnitude diagram for the intermediate-age star clusters.
\end{abstract}

Key words: stars: evolution — stars: rotation — stars: interiors

\section{INTRODUCTION}

Recently, it has been discovered that some intermediate-age star clusters have a double or extended main-sequence turnoffs (MSTOs) in their color-magnitude diagrams (CMDs) (Mackey \& Broby Nielsen 2007, Mackey et al. 2008, Milone 2009, Goudfrooij et al. 2009, 2011). Platais et al. (2012) also found that the upper main sequence of the open cluster Trumpler 20 with an age of about $1.3 \mathrm{Gyr}$ appears to show an enlarged color spread which is not normally seen in the CMD for open clusters in this age group. The double or extended MSTOs are interpreted as that the star clusters have two or multiple stellar populations with similar metal abundance but with differences in age of $\sim 200$ - $300 \mathrm{Myr}$ (Mackey et al. 2008), which is contrary to the traditional knowledge that a star cluster is comprised of stars belonging to a single, simple stellar population with a uniform age and chemical composition. However, Platais et al. (2012) also pointed out that the enlarged color spread of Trumpler 20 may be due to differential reddening. In order to understand the extended MSTO, many scenarios were proposed by many investigators (Mackey \& Broby Nielsen 2007, Bekki \& Mackey 2009, Goudfrooij et al. 2009. Bastian \& de Mink 2009, Rubele et al. 2010, 2011, Yang et al. 2011b, Girardi et al. 2011). In these scenarios, Yang et al. (2011b) found that the interactions in binary systems can reproduce an extended 
MSTO of star clusters. However, the fraction of the interactive binary systems is too low to completely explain the observed features in star clusters. Another interpretation proposed by Rubele et al. (2010, 2011) and Girardi et al. (2011) is continuous star formation, lasting 300 Myr or longer. However, Platais et al. (2012) noted that 'this long period of star formation seems to be odds with the fact that none of the younger clusters are known to have such a trait', and that some star clusters with extended MSTO might not experience a self-enrichment. Yang et al. (2011b) also noted that the lasting time for explaining the double MSTO and dual red clump of a star cluster is not consistent.

Rotation is a property that virtually all stars possess. Observations show that main-sequence (MS) stars with $1.3<\mathrm{M} / \mathrm{M}_{\odot}<3.0$ have a typical value of $160 \mathrm{~km} \mathrm{~s}^{-1}$ of $v \sin i$ (Royer et al. 2007) which corresponds to a period of $\sim 0.5-0.8$ day. In the classical theory of stellar evolution, the effects of rotation on stellar evolutions are always neglected. However, in fact, rotation is one of the key factors that can change the evolution and all outputs of stellar models (Maeder \& Meynet 2000). Bastian \& de Mink (2009) calculated the evolutions of rotating models with an initial mass of $1.5 \mathrm{M}_{\odot}$ but with different rotation rates using the stellar evolution code described by Yoon et al. (2006) in which the effects of rotation on the structure and mixing induced by rotation are taken into account. They found that rotating models are cooler and fainter than non-rotating ones for not extreme rotation rates. They incorporated these effects of rotation on stellar evolutions into the sythesis of CMD of star clusters. They found that stellar rotations can mimic the effect of multiple populations in star clusters, whereas in actuality only a single population exists. However, using the Geneva stellar evolution code, Eggenberger et al. (2010) and Girardi et al. (2011) calculated the evolutions of rotating models with an initial velocity on the zeroage main sequence (ZAMS) of $150 \mathrm{~km} \mathrm{~s}^{-1}$, they found that rotating models can be slightly hotter and brighter than non-rotating ones. The isochrone of rotating stars has a slightly hotter and brighter turnoff with respect to that of non-rotating stars (Girardi et al.2011), which is contrary to the calculation result of Bastian \& de Mink (2009). The origin of the extended MSTO of intermediate-age star clusters is still an open question. In order to understand the effects of stellar rotation on the evolutions of stars and on the CMD of star clusters, the evolutions of rotating stars need to be studied in more detailed.

In this paper, we mainly focus on the effects of rotation on the evolutions of stars, especially on the evolutions of the intermediate-mass MS stars which corresponds to the MSTO stars of intermediate-age star clusters. The paper is organized as follows: we give a physical description of the effects of rotation in Section 2, present the calculation results in Section 3 and discuss and summarize them in Section 4.

\section{PHYSICAL DESCRIPTION OF ROTATION}

\subsection{Effects of rotation on stellar structure and evolution}

The equations of stellar structure of a rotating star were built up by Kippenhahn \& Thomas (1970) and modified by Endal \& Sofia (1976) and Meynet \& Maeder (1997) to calculate shellular rotation. Rotation affects stellar structure and evolution mainly in four ways:

1. The first one is the effect of centrifugal force on the effective gravity. This effect can be considered directly in the equation of hydrostatic equilibrium.

2. The second is that the equipotential or isobar surfaces are no longer spheres for a rotating star because the centrifugal force is always perpendicular to the axis of rotation and is not, in general, parallel to the force of gravity. Thus the assumption of spherical symmetry for non-rotating stars is no longer fit for rotating ones. A rotating star may be a spheroid. This leads to the fact that almost all of structure equations are affected and the independent variable of equations of stellar structure need to be redefined.

3. The third is that the radiative flux is not constant on an isobar surface because the radiative flux varies with the local effective gravity, i.e. the Von Zeipel effect (Von Zeipel 1924), which affects the radiative equilibrium equation and the instability of convection by changing the radiative temperature gradient.

4. The final results from the transport of angular momentum and the mixing of elements caused by rotationally induced instabilities. The mixing happening in the stellar interiors can affect the density 
Table 1 The values of the parameters for angular momentum loss, the transport of angular momentum, and element mixing.

\begin{tabular}{cccccc}
\hline Parameter & $f_{k}$ & $\beta$ & $\Omega_{\text {crit }}$ & $f_{\Omega}$ & $f_{c}$ \\
\hline Value & $1.0^{a}\left(2.5^{b}\right)$ & 1.5 & $5 \Omega_{\odot}$ & 1.0 & 0.2 \\
\hline
\end{tabular}

Note.- $a$ for models with $M>1.0 \mathrm{M}_{\odot} ; b$ for $1.0 \mathrm{M}_{\odot}$ model.

distribution by changing the mean molecular weight. Thus it can affect the radius of stars, the instability of convection, and so on.

The first three effects are directly incorporated into the equations of stellar structure (Endal \& Sofia 1976). In a nonrotating model the independent variable, $M_{r}$, is the mass contained within a spherical surface. However, in the rotating one the independent variable, $M_{p}$, is the mass contained within an isobar surface. The algorithmic methods of the isobar surface and physical quantities on the isobar surface can be found in Endal \& Sofia (1976), Meynet \& Maeder (1997), and Yang \& Bi (2006) in detail.

\subsection{Angular momentum loss, angular momentum transport and the mixing of elements}

In this context, we assumed that angular momentum loss is caused by magnetic stellar winds and the loss happens only when the stellar envelope is convective. The parameterized Kawaler's relations (Kawaler 1988, Chaboyer et al.1995)

$$
\begin{gathered}
\frac{d J}{d t}=f_{k} K_{w}{\frac{R}{R_{\odot}}}^{2-\beta}\left(\frac{M}{M_{\odot}}\right)^{-\beta / 3}\left(\frac{\dot{M}}{10^{-14}}\right)^{1-2 \beta / 3} \Omega^{1+4 \beta / 3} \quad\left(\Omega<\Omega_{c r i t}\right), \\
\frac{d J}{d t}=f_{k} K_{w}\left(\frac{R}{R_{\odot}}\right)^{2-\beta}\left(\frac{M}{M_{\odot}}\right)^{-\beta / 3}\left(\frac{\dot{M}}{10^{-14}}\right)^{1-2 \beta / 3} \Omega \Omega_{c r i t}^{4 \beta / 3} \quad\left(\Omega \geq \Omega_{c r i t}\right)
\end{gathered}
$$

were used to calculate the rate of angular momentum loss, where $f_{k}, \beta$, and $\Omega_{\text {crit }}$ are adjustable parameters, $K_{w}=2.035 \times 10^{33} \times\left(1.442 \times 10^{9}\right)^{\beta}$ in cgs units, the mass-loss rate $\dot{M}$ is set to $2.0 \times 10^{-14} \mathrm{M}_{\odot}$ $\mathrm{yr}^{-1}$. The value of $f_{k}$ calibrated to the Sun is 2.5 , however, which may overestimate the rate of angular momentum loss for stars with mass larger than the Sun because these stars have a shallower convective envelope than the Sun. Thus we adopted a value of 1.0 for the stars with mass larger than the Sun.

In addition, we assumed that the rotation is uniform on the ZAMS and in all convective regions of a star. The angular momentum loss, expansion and/or contraction of stars lead to the occurrence of differential rotation in radiative regions. The rotational instabilities induced by rotation not only can transport angular momentum but also can mix chemical elements. Because the horizontal turbulent is stronger than the vertical turbulent, the angular velocity and chemical compositions are constant on an isobar (Zahn 1992). Thus we only consider the vertical transports of angular momentum and chemical elements which obey two coupled nonlinear diffusion equations:

$$
\rho r^{2} \frac{I}{M} \frac{\partial \Omega}{\partial t}=f_{\Omega} \frac{\partial}{\partial r}\left(\rho r^{2} \frac{I}{M} D \frac{\partial \Omega}{\partial r}\right)-\frac{\partial}{\partial r}\left(\rho r^{2} \frac{I}{M} \Omega \dot{r}\right)
$$

for the transport of angular momentum and

$$
\frac{\partial X_{i}}{\partial t}=\left(\frac{\partial X_{i}}{\partial t}\right)_{n u c}-\frac{1}{\rho r^{2}} \frac{\partial}{\partial r}\left(\rho r^{2} X_{i} V_{i}\right)+f_{\Omega} \frac{1}{\rho r^{2}} \frac{\partial}{\partial r}\left(\rho r^{2} f_{c} D \frac{\partial X_{i}}{\partial r}\right)
$$

for the change in the the mass fraction $X_{i}$ of species $i$, where the fraction $I / M$ is the momentum of inertia per unit mass, and $D$ is the diffusion coefficient due to the rotational instabilities including meridional circulation, the Goldreich-Schubert-Fricke instability (Goldreich \& Schubert 1967, Fricke 1968), 


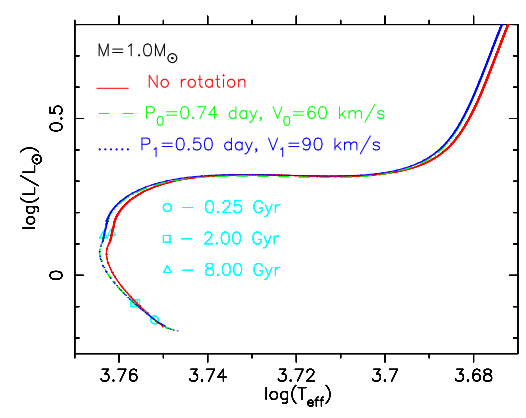

Fig. 1 Evolutionary tracks in the Hertzsprung-Russell (HR) diagram for $1 \mathrm{M}_{\odot}$ models. The solid (red) line corresponds to the track of the non-rotating model, the dashed (green) and dotted (blue) lines show that of the rotating models with an initial period of 0.74 and 0.50 day, respectively. The positions of models at the ages of $0.25,2.0$, and $8.0 \mathrm{Gyr}$ are marked on the tracks.

and the secular shear instability. The criterion and the estimate of the coefficient $D$ of these instabilities were summarized by Endal \& Sofia (1978) and Pinsonneault et al. (1989) in detail. The second term on the right-side of Eq. (3) describes the change of angular velocity induced by contraction and/or expansion of stars. The first and second term on the right-side of Eq. (4) is due to the nuclear reaction and gravity settling diffusion, respectively. The velocity, $V_{i}$, of the gravity settling diffusion is given by Thoul et al. (1994). The adjustable parameter $f_{\Omega}$ is introduced to represent some inherent uncertainties in the diffusion equation, and the $f_{c}$ is used to account for the fact that the transports of angular momentum and a chemical species occur on different timescales and is less than one. The parameters $f_{\Omega}$ and $f_{c}$ may depend on stellar mass. However, for simplicity, they are assumed to be a constant. The values of the adjustable parameters in Eqs. (1)-(4) are summarized in Table11 The effects of magnetic fields in the stellar interiors are not considered in our models. The magnetic fields are introduced to explain the flat rotational profile in the Sun (Brown et al. 1989, Kosovichev et al. 1997, Eggenberger et al. 2005, Yang \& $\mathrm{Bi} 2006$, 2008). However, the gravity waves also can be competent to explain the solar rotation (Zahn et al. [1997, Talon \& Charbonnel[2005). Especially for the intermediate-mass stars which do not experience magnetic braking, our calculations show that the rotational instabilities are efficent enough to transport angular momentum in these stars.

\section{RESULTS}

We used the Yale Rotation Evolution Code (YREC7) to compute the evolutions of rotating and nonrotating models with masses between 1.0 and $3.0 \mathrm{M}_{\odot}$. The code has been updated with recent input physics over the last three decades (Endal \& Sofia 1976, 1978, Pinsonneault et al. 1989, Chaboyer et al. 1995, Yang \& Bi 2007). The new OPAL EOS tables (Rogers \& Nayfonov 2002), OPAL opacity tables (Iglesias \& Rogers [1996), and the opacity tables for low temperature provided by Alexander \& Ferguson (1994) were used. Energy transfer by convection is treated according to the standard mixing length theory. The value of 1.72 for the mixing-length parameter $(\alpha)$ was calibrated against the Sun. The initial chemical compositions of our models are fixed at $Z_{0}=0.02$ and $X_{0}=0.707$. The initial rotation period $P_{0}$ (or initial rotation velocity $V_{0}$ ) is a free parameter. We mainly computed the evolutions of the models with $P_{0}=0.74$ and 0.50 day, respectively. All models share the same initial parameters except for mass and rotation period. 

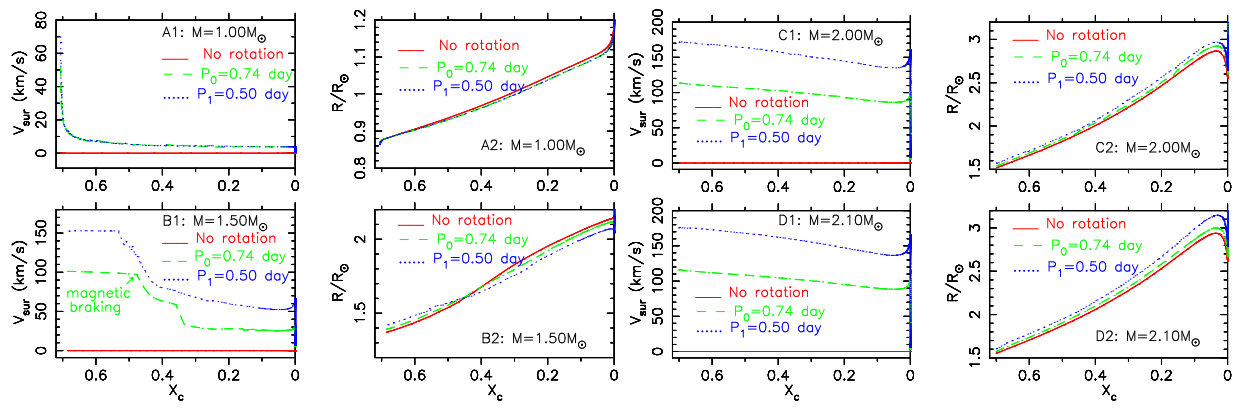

Fig. 2 The surface (equatorial) velocity and radius as a function of the mass fraction of central hydrogen.The solid (red) line corresponds to the non-rotating model, the dashed (green) and dotted (blue) lines indicate the rotating models with an initial period of 0.74 and 0.50 day, respectively.

\subsection{For the stars with mass less than $1.5 \mathrm{M}_{\odot}$}

Figure 1 1 shows the evolutionary tracks of the $1.0 \mathrm{M}_{\odot}$ models with and without rotation. No matter what the initial rotation period, the efficient magnetic braking almost results in the same rotational velocity (see the panel A1 in Fig. 22) within a few hundred Myr, which leads to the fact that the evolutionary tracks of the two rotating models are almost overlapped in the Hertzsprung-Russell (HR) diagram. When the age of stars is younger than about $0.25 \mathrm{Gyr}$, the effective temperature and luminosity of the rotating models are slightly lower than that of the non-rotating ones. However, when age $>0.25 \mathrm{Gyr}$, the effective temperature of the rotating models is higher but luminosity is lower than that of the non-rotating one at the same age.

The rotational mixing takes into effect only after composition gradients have been produced. When age $<0.25 \mathrm{Gyr}$, the variation of chemical compositions with respect to radius is very small for a 1.0 $\mathrm{M}_{\odot}$ model, thus the effects of rotational mixing can be neglected and the hydrostatic effects of rotation play a dominant role in this stage. The hydrostatic effects of rotation result in a decrease in the effective gravity of stars, which can slightly decrease the central temperature and enlarge the radius of rotating models compared to non-rotating ones. Therefore, the rotating models have a slightly lower luminosity and effective temperature than non-rotating ones.

When age $>0.25 \mathrm{Gyr}$ (the central hydrogen abundance $X_{c} \lesssim 0.69$ ), due to the angular momentum loss, on the one hand, the rotation rate becomes low enough to neglect the influences of the hydrostatic effects on the radius; on the other hand, the increase in the gradients of angular velocity and chemical compositions with respect to radius makes that the rotational mixing begins to play a dominant role by feeding more fresh hydrogen fuel into the hydrogen-burning region and by transporting helium outwards, which can lead to an increase in the mean density, i.e. decrease in radius, and deceleration of evolution. Thus the rotating models exhibit a higher central hydrogen abundance (see the panel A in Fig. 3) and effective temperature but slightly lower luminosity than non-rotating ones at the same age. When the models evolved to the same evolutionary state (the same central hydrogen abundance), the luminosity of rotating models is only slightly higher than that of non-rotating ones by consuming more hydrogen, however the radius of rotating models is still smaller than that of non-rotating ones (see the panel A2 in Fig. 2) due to the effects of the rotational mixing, therefore the effective temperature of rotating models is higher than that of non-rotating ones (see panels A1 and A2 in Fig. (4). If the rotational mixing is not considered, Fig. 5 shows that rotation almost not affects the evolution of a $1.0 \mathrm{M}_{\odot}$ model after the age of $0.25 \mathrm{Gyr}$.

In Fig. 6, we show the evolutionary tracks of rotating and non-rotating models with $M=1.2$ and 1.4 $\mathrm{M}_{\odot}$. The effects of rotation on the evolutions of these stars are similar to those on the evolution of the $1.0 \mathrm{M}_{\odot}$ model. Compared with the tracks of non-rotating models, the tracks of rotating models 

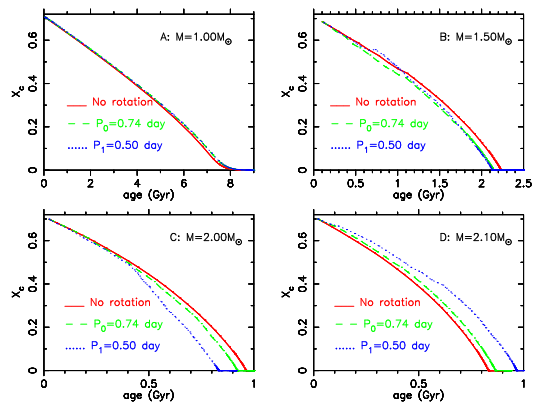

Fig. 3 The mass fraction of central hydrogen as a function of age. The solid (red) line corresponds to the non-rotating model, the dashed (green) and dotted (blue) lines show the rotating models with an initial period of 0.74 and 0.50 day, respectively.

move to the left of the HR diagram. For stars with $M \lesssim 1.4 \mathrm{M}_{\odot}$, due to the efficient magnetic braking, the hydrostatic effects of rotation play a dominant role only in about $100 \mathrm{Myr}$. Then, just as the case of $1.0 \mathrm{M}_{\odot}$ models, the rotational mixing begins to play a dominant role. The rotating models exhibit a higher effective temperature and an approximately equal luminosity compared to the non-rotating ones when they evolved into the same evolutionary state [for instance the beginning of the MS hook (Yang et al. 2011a) in the HR diagram]. Moreover, for the star with $M=1.4 \mathrm{M}_{\odot}$, Fig. 6 displays that the rotating models have higher effective temperatures and luminosities at the age of 1.0 Gyr but have lower effective temperatures at the age of $1.75 \mathrm{Gyr}$ than non-rotating ones, and that the rotating models evolve faster than the non-rotating ones, which are different from the evolutions of the models with $M=1.0$ $\mathrm{M}_{\odot}$.

The radiative temperature gradient can be changed by Von Zeipel (1924) effect, while the adiabatic temperature gradient can be influenced by the mixing of elements caused by hydrostatic instabilities. Thus the instability of convection can be affected by the effects of rotation. Our calculations show that the effects of rotation can result in a decrease in the radius or mass of the convective core for stars with $1.1 \mathrm{M}_{\odot} \lesssim M<2.05 \mathrm{M}_{\odot}$ but an increase for stars with $M>2.05 \mathrm{M}_{\odot}$ (see Fig. 7 ). The decrease in the convective core leads to the fact that the hydrogen abundance in the convective core of rotating models decreases faster than that of non-rotating ones. Although the rotational mixing can bring hydrogen fuel into the core from outer layers, which can not compensate the decrease of the central hydrogen caused by the decrease in the convective core. As a consequence, the rotating models evolve faster than nonrotating ones during the MS stage for stars with $1.1 \mathrm{M}_{\odot} \lesssim M \lesssim 2.0 \mathrm{M}_{\odot}$ (see Fig. 3). Furthermore, Fig. 7 shows that the faster the rotation, the smaller the convective core, thus the faster the evolution of the MS stage for stars with $1.1 \mathrm{M}_{\odot} \lesssim M \lesssim 2.0 \mathrm{M}_{\odot}$. We labelled this phenomenon as 'rotation acceleration'. The rotation acceleration leads to the fact that the rotating models with $M=1.4 \mathrm{M}_{\odot}$ exhibit the higher luminosities at the age of $1.0 \mathrm{Gyr}$ and lower effective temperatures at the age of 1.75 Gyr than the non-rotating ones (see Fig. 6).

\subsection{For the stars with masses between 1.5 and $1.7 \mathrm{M}_{\odot}$}

Figure 8 shows the evolutionary tracks of the rotating and non-rotating models with $M=1.5,1.6$, and $1.7 \mathrm{M}_{\odot}$. For stars with $M \gtrsim 1.5 \mathrm{M}_{\odot}$, their envelopes are radiative at the beginning of MS. Before the appearance of convective envelope (i.e. before magnetic braking), the stars maintain a fast rotation (see the panel B1 in Fig. 2), the hydrostatic effects of rotation dominate the corrections to models and lead to the rotating models having a larger radius (see the panel B2 in Fig. 2) and slightly lower central temperature than the non-rotating ones, thus the rotating models have a slightly lower luminosity and effective temperature than the non-rotating ones at the same evolutionary stage in the early stage of MS 

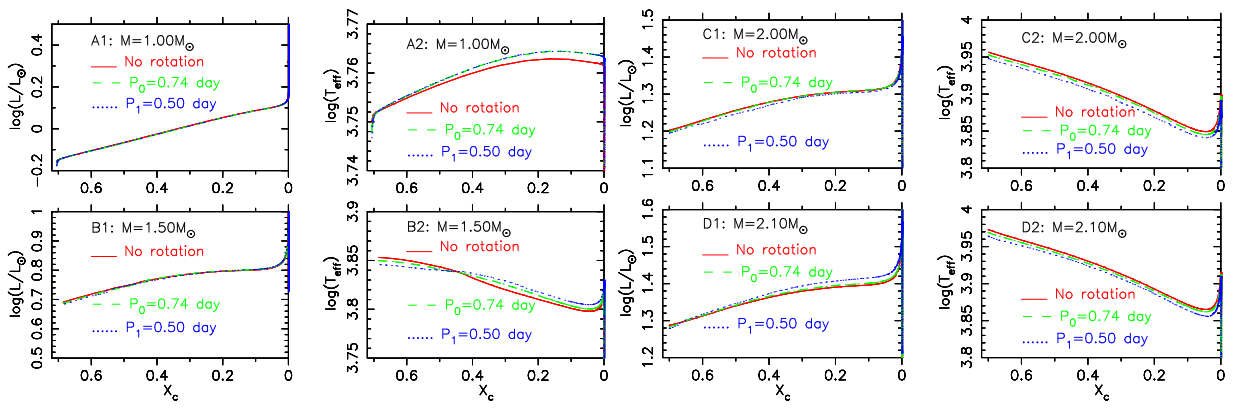

Fig. 4 The luminosity and effective temperature as a function of the mass fraction of central hydrogen.The solid (red) line corresponds to the non-rotating model, the dashed (green) and dotted (blue) lines indicate the rotating models with an initial period of 0.74 and 0.50 day, respectively.

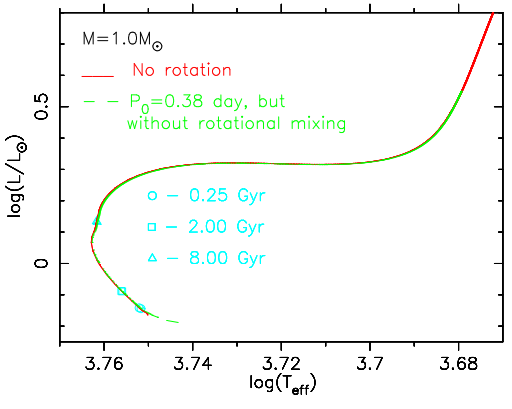

Fig. 5 Same as Fig 1 but the rotating model without including the effects of rotational mixing.
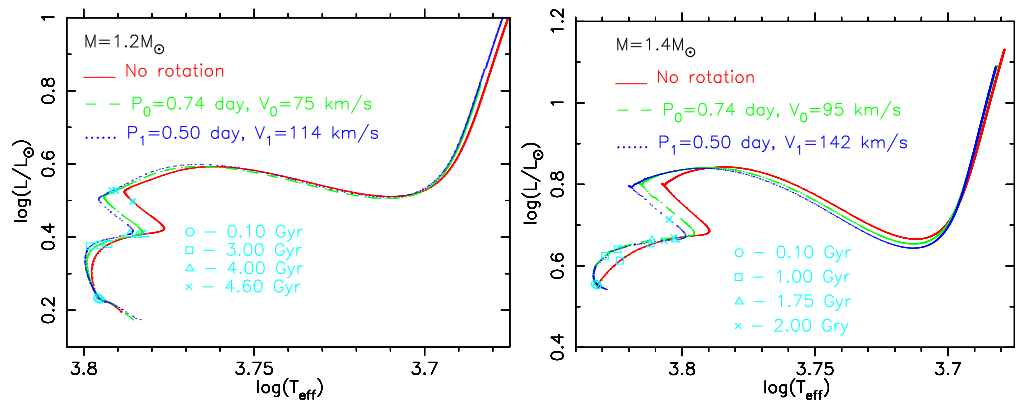

Fig. 6 Same as Fig 1 but for stars with $\mathrm{M}=1.2$ and $1.4 \mathrm{M}_{\odot}$, respectively.

(see the panels B1 and B2 in Fig. (4). However due to the effects of the rotation acceleration, as the evolution proceeds, the rotating models can exhibit a slightly higher luminosity than non-rotating one at the same age.

After the convective envelope has developed, due to the angular momentum loss, on the one hand, the rotation of the stars begins to become slow, which results in a reduction in the hydrostatic effects of rotation (for example stellar radius is almost no longer affected by the hydrostatic effects of rotation); 

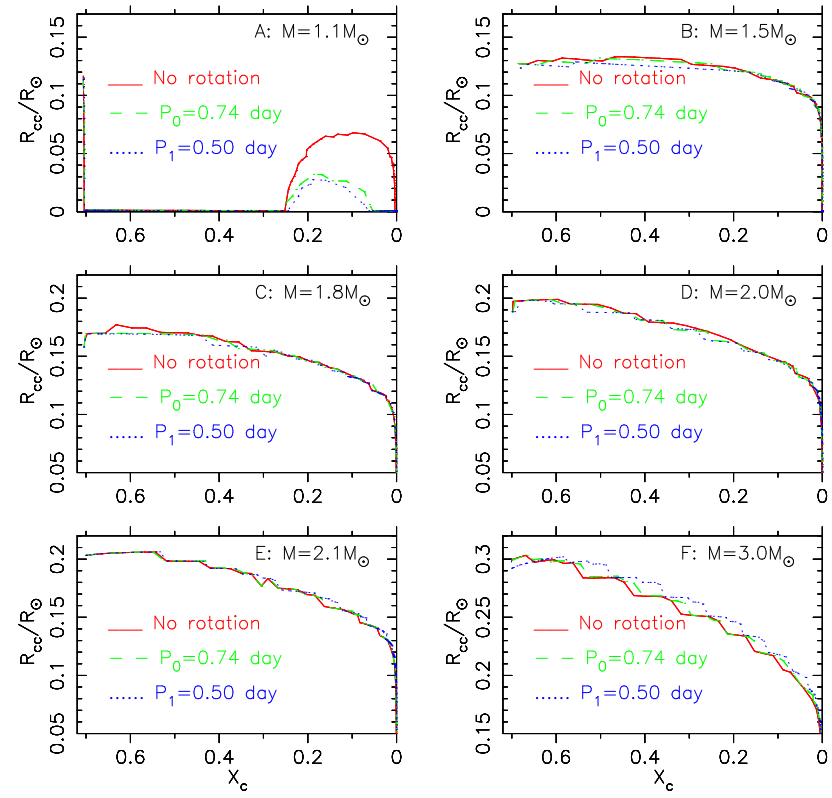

Fig. 7 The radius of convective core as a function of the mass fraction of central hydrogen abundance. The solid (red) line corresponds to the non-rotating model, the dashed (green) and dotted (blue) lines indicate the rotating models with an initial period of 0.74 and 0.50 day, respectively.

on the other hand, the gradient of angular velocity with respect to radius increases, which enhances the effects of the rotational mixing. As the evolution proceeds, the rotational mixing begins to play a dominant role, thus the radius of rotating models changes from larger to smaller than that of non-rotating ones (see the panel B2 in Fig. 22). In addition, when the models with and without rotation evolved to the same evolutionary state of MS stage, their luminosities are approximately equal, therefore the effective temperatures of rotating models change from lower to higher than that of non-rotating ones (see the panel B2 in Fig. (4). However, before the MS hook of stars with $M \gtrsim 1.6 \mathrm{M}_{\odot}$, due to the facts that the magnetic braking occurs in the late stage of the MS and the rotation accelerates the evolutions of rotating models, the effective temperature of rotating models is always lower than that of non-rotating ones at the same age. For example, at the age of $1.5 \mathrm{Gyr}$, the effective temperature of the star with $M=$ $1.6 \mathrm{M} \odot$ is about $6620 \mathrm{~K}$ for the non-rotating model but is $6540 \mathrm{~K}$ for the rotating model with $T_{0}=0.74$ day. The difference in the effective temperatures is $80 \mathrm{~K}$.

Moreover, Fig. 8 shows that the luminosities of rotating models are slightly higher than that of nonrotating one at the end of MS hook. This is because rotation hardly affects the convective core when $X_{c}<0.1$ for these stars and the rotational mixing makes rotating models consuming slightly more hydrogen than non-rotating ones during the MS hook.

\subsection{For the Stars with masses between 1.8 and $2.0 \mathrm{M}_{\odot}$}

The evolutionary tracks of the rotating and non-rotating models with $M=1.8$ and $2.0 \mathrm{M}_{\odot}$ are shown in Fig. 9 For these stars, the rotating models almost exhibit lower luminosities and effective temperatures than non-rotating ones when they reached the same evolutionary state in the whole MS stage (see the panels $\mathrm{C} 1$ and $\mathrm{C} 2$ in Fig. (4). Before the MS hook, even at the same age the effective temperatures of the rotating models are also lower than that of non-rotating ones, but their luminosities are approximately equal. For example, for the star with $M=1.8 \mathrm{M}_{\odot}$, at the age of $1.1 \mathrm{Gyr}$ the effective temperature and 


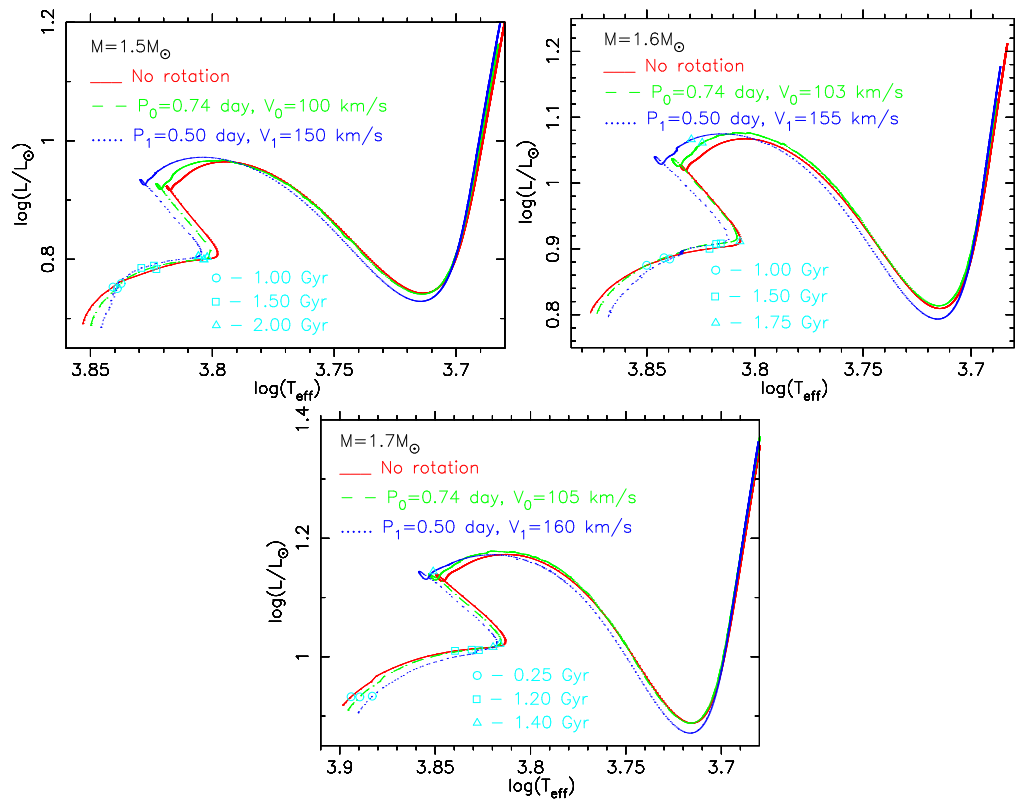

Fig. 8 Same as Fig 1 but for stars with $\mathrm{M}=1.5,1.6$, and $1.7 \mathrm{M}_{\odot}$, respectively.
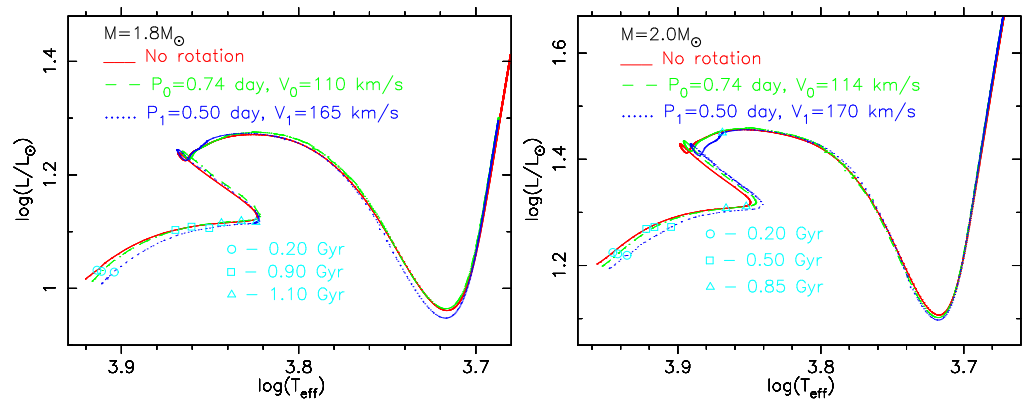

Fig. 9 Same as Fig 1 but for the models with $M=1.8$ and $2.0 \mathrm{M}_{\odot}$, respectively.

luminosity is $6970 \mathrm{~K}$ and $13.03 L_{\odot}$ for the non-rotating model but $6670 \mathrm{~K}$ and $13.08 L_{\odot}$ for rotating model with $T_{0}=0.5$ day, respectively. The difference in the effective temperatures is about $300 \mathrm{~K}$. This is because these stars do not experience magnetic braking during the MS stage. The fast rotation leads to the fact that the hydrostatic effects of rotation dominate the corrections to radius. In addition, the effects of the mixing caused by rotationally induced instabilities are partly counteracted by the effect of the decrease in the convective core in these models. Thus the rotating models have a larger radius than non-rotating ones at the same evolutionary state (As a case, see the panel C2 in Fig. 22). Moreover, the hydrostatic effects of fast rotation also leads to a decrease in the central temperature. For example, when the star with $M=2.0 \mathrm{M}_{\odot}$ evolved to $X_{c}=0.2607$, the central temperature is $2.2978 \times 10^{7} K$ for the non-rotating model but is $2.2966 \times 10^{7} \mathrm{~K}$ for the rotating model with $P_{0}=0.5$ day. The lower the central temperature, the lower the energy product of $\mathrm{H}$-burning. Thus the rotating models have slightly lower luminosities than non-rotating ones at the same evolutionary state. As a consequence, the effective temperatures of rotating models are lower than those of non-rotating ones. 

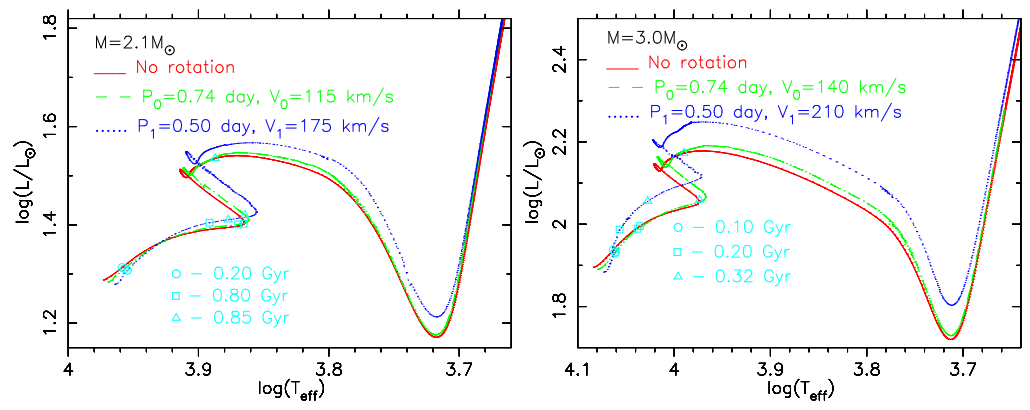

Fig. 10 Same as Fig 1 but for the models with $\mathrm{M}=2.1$ and $3.0 \mathrm{M}_{\odot}$, respectively.

\subsection{For the stars with mass larger than $2.1 \mathrm{M}_{\odot}$}

In Fig. 10, we show the evolutionary tracks of the 2.1 and $3.0 \mathrm{M}_{\odot}$ models with and without rotation. For the stars with $M \geq 2.1 \mathrm{M}_{\odot}$, in the early stage of the MS (the $X_{c}>0.5$ for $M=2.1 \mathrm{M}_{\odot}$ and $X_{c}>$ 0.6 for $M=3.0 \mathrm{M}_{\odot}$ ) the hydrostatic effects of rotation dominate the corrections to stellar models; thus the rotating models exhibit lower luminosities and effective temperatures than the non-rotating ones at the same age.

As the evolution proceeds, the effects of rotation leads to an increase in the convective core for these stars, and the more massive the star or the higher the rotation rate, the larger the change of the convective core (see Fig. 77). Both the increase of the convective core and rotational mixing can enhance the hydrogen abundance and decrease the helium abundance in the core, which leads to an increase in the mean density (i.e. a decrease in radius) and decrease in the central temperature of the rotating models compared to non-rotating ones at the same age, and prolongs the lifetime of core $\mathrm{H}$ burning. In addition, the hydrostatic effects of rotation also can lead to a decrease in the central temperature. Thus rotating models evolve slower than non-rotating ones and exhibit lower luminosities than non-rotating ones at the same age. The effects of rotation result in an increase in the convective core for stars with $M \gtrsim$ $2.1 \mathrm{M}_{\odot}$ but to a decrease in the convective core for stars with $M \lesssim 2.0 \mathrm{M}_{\odot}$. Chemical elements are mixed completely in the convective cores. The increase of the convective core causes that the location of the chemical element gradient moves outwards, which leads to the facts that the product of H-burning can be much more efficiently transported outwards by rotational mixing. Thus the rotationally induced element mixing in stars with $M \gtrsim 2.1 \mathrm{M}_{\odot}$ is more efficient than that in stars with mass between about 1.7 and $2.0 \mathrm{M}_{\odot}$. For example, when the stars with $M=2.0$ and $2.1 \mathrm{M}_{\odot}$ evolved to $X_{c}=0.261$, the surface hydrogen abundance is 0.707 for both non-rotating models, however it is 0.705 for the rotating model with $M=2.0 \mathrm{M}_{\odot}$ and $P_{0}=0.5$ day but is 0.700 for the rotating model with $M=2.1 \mathrm{M}_{\odot}$ and $P_{0}=0.5$ day. The efficient mixing leads to the fact that the radius of the rotating models is smaller than that of non-rotating ones at the same age and the change of the radius is larger that of luminosity. Thus the rotating models exhibit the higher effective temperatures than non-rotating ones at the same age during the middle stage of MS. However, when the models evolved into the same evolutionary state of the late stage of MS, because the rotating models consumed more hydrogen fuel than non-rotating ones, which can enhance the temperatures of core and the He-core mass left behind, the rotating models have a larger energy product of $\mathrm{H}$ burning than non-rotating ones. The higher the energy product, the more the expansion of stars. Thus the rotating models exhibit larger radii and lower effective temperatures than non-rotating ones at the same evolutionary state. The effects of rotation on the evolutions of these stars with $M \gtrsim 2.1 \mathrm{M}_{\odot}$ are similar to the effects of convective core overshooting. 


\section{DISCUSSION AND CONCLUSIONS}

Besides the influences of rotation on the internal evolution of stars, effects of rotation on the observable parameters of stars depend on the product $v \sin i$, where $v$ is the equatorial rotational velocity and $i$ refers to the angle between the rotational axis of a star and the direction towards the observor. In this work, we only focus on the effects on the internal stellar evolution.

The effects of rotation on stellar structure and evolution mainly derive from the hydrostatic effects, the mixing of elements caused by rotationally induced instabilities, and the Von Zeipel effect which affects the instability of convection by changing the radiative temperature gradient. The hydrostatic effects mainly lead to an increase in radius and a decrease in the effective temperature. The mixing of elements, however, chiefly results in an increase in the mean density, i.e. a decrease in radius and an increase in the effective temperature. Moreover, rotation leads to a decrease in the convective core for stars with $M<2.05 \mathrm{M}_{\odot}$, which can counteract the effects of the rotational mixing and accelerate the evolution of stars. However, rotation results in an increase in the convective core for stars with $M>$ $2.05 \mathrm{M}_{\odot}$, which dominates the effect of rotation on the evolutions of these stars. For the models with masses between about 1.7 and $2.0 \mathrm{M}_{\odot}$, because the effects of the rotational mixing is counteracted by the effects of the decrease in the convective core and they do not experience magnetic braking during the MS stage, the hydrostatic effects dominate the effects on the effective temperatures and luminosities of these models; thus, the effective temperatures and luminosities of rotating models are lower than those of non-rotating ones during the MS stage.

The evolutions of our rotating models with $M \gtrsim 2.1 \mathrm{M}_{\odot}$ are consistent with the calculation results of Eggenberger et al. (2010) and Girardi et al. (2011). However the rotating models with mass between $\sim 1.7$ and $2.0 \mathrm{M}_{\odot}$ manifest lower effective temperatures than non-rotating ones, which is not consistent with the results of Girardi et al. (2011) but is similar to the calculation result of Bastian \& de Mink (2009). Moreover, in our models, the MS bandwidth of rotating models with $M>2.0 \mathrm{M}_{\odot}$ is wider than that of non-rotating ones. However, the MS bandwidth of rotating models with $M<2.0 \mathrm{M}_{\odot}$ is narrower than that of non-rotating ones, which is consistent with the distributions of the large sample of rotating stars collected by Royer et al. (2007) in the HR diagram (see the Fig. 4 of Zorec \& Royer 2012). In the next work, we will give a more detailed comparison.

For MS stars with a given rotation rate, the change in the effective temperature caused by rotation increases with increasing mass. For example, when age $=1.1 \mathrm{Gyr}$ and $P_{0}=0.5$ day, the difference of the effective temperature between non-rotating and rotating model is about $300 \mathrm{~K}$ for the stars with mass between 1.7 and $1.8 \mathrm{M}_{\odot}$, is around $200 \mathrm{~K}$ for the star with $M=1.6 \mathrm{M}_{\odot}$, and is about $40 \mathrm{~K}$ for the star with $M=1.5 \mathrm{M}_{\odot}$, but is only several Kelvin for the stars with $M \lesssim 1.4 \mathrm{M}_{\odot}$. The change in the effective temperature caused by rotation decreases with decreasing mass. Thus, for some intermediateage star clusters, rotation might lead to a color spread near the MSTO in their CMD. In the next work, we will give and discuss the isochrone of rotating models.

In this work, we calculated a grid of evolutionary tracks of rotating models with masses between 1.0 and $3.0 \mathrm{M}_{\odot}$ and a resolution $\delta M \leq 0.02 \mathrm{M}_{\odot}$. We find that the effects of rotation on stellar structure and evolution are dependent not only on the rotation rate but also on the mass of stars. For stars with $M>$ $2.05 \mathrm{M}_{\odot}$, rotation leads to an increase in the convective core and prolongs the lifetime of core $\mathrm{H}$ burning; the evolution of rotating models of these stars is slower than that of non-rotating ones; in the early stage of MS, the changes in luminosities and effective temperatures are mainly due to the hydrostatic effects of rotation, thus rotating models exhibit lower luminosities and effective temperatures than non-rotating ones at the same age; however, in the late stage of MS, rotating model can manifest higher effective temperatures than non-rotating ones at the same age and larger luminosities at the same evolutionary stage because the rotational mixing dominates the effects on the models. For stars with $1.1 \mathrm{M}_{\odot} \lesssim M<$ $2.05 \mathrm{M}_{\odot}$, rotation results in a decrease in the convective core and shortens the lifetime of core $\mathrm{H}$ burning; the rotating models of these stars evolve faster than non-rotating ones. When $1.7 \mathrm{M}_{\odot} \lesssim M<2.05 \mathrm{M}_{\odot}$, the rotating models exhibit lower effective temperatures but approximately equal luminosities compared to non-rotating ones at the same age; the evolutionary tracks of the rotating models are located on the lower right of that of non-rotating ones in the HR diagram, which are mainly due to the hydrostatic 
effects of rotation and 'rotation acceleration'. However, for stars with $1.0 \mathrm{M}_{\odot} \lesssim M \lesssim 1.4 \mathrm{M}_{\odot}$, due to the fact that these stars experienced the magnetic braking from the beginning of evolution, the rotational mixing and 'rotation acceleration' dominate the effects of rotation on the evolution of these stars; the evolutionary tracks of rotating models of these stars are mainly located on the left of that of non-rotating ones in the HR diagram; the rotating models can exhibit lower or higher effective temperatures than non-rotating ones at the same age, which depends on the mass and age of stars. Our calculations show that the mass of $2.05 \mathrm{M}_{\odot}$ is a critical value for the effect of rotation on the stellar structure and evolution. This value is very close to the critical mass $\left(2.01 \mathrm{M}_{\odot}\right)$ for oscillations of horizontal branch stars (Yang et al. 2012). Rotation could lead to a color spread for some intermediate-age star clusters near the MSTO in their CMD.

Acknowledgements This work was supported by China Postdoctoral Science Foundation through grant 20100480222, the NSFC through grants 11273012, 11273007, 10773003, 10933002, and 11003003, the Ministry of Science and Technology of the People's republic of China through grant 2007CB815406, and the Project of Science and Technology from the Ministry of Education (211102).

\section{References}

Alexander D. R., \& Ferguson J. W., 1994, ApJ, 437, 846

Bastian N., \& de Mink S. E., 2009, MNRAS, 398, L11

Bekki K., \& Mackey A. D., 2009, MNRAS, 394, 124

Brown T. M., Christensen-Dalsgaard J., Dziembowski W. A., Goode P., Gough D. O., \& Morrow C. A., 1989, ApJ, 343,526

Chaboyer B., Demarque P., \& Pinsonneault M. H., 1995, ApJ, 441, 865

Endal A. S., \& Sofia S., 1976, ApJ, 210, 184

Endal A. S., \& Sofia S., 1978, ApJ, 220, 279

Eggenberger P., Maeder A., \& Meynet G., 2005, A\&A, 440, L9

Eggenberger P., Miglio A., Montalban J., Moreira O., Noels A., Meynet G., \& Maeder A., 2010, A\&A, 509 , A72

Fricke K., 1968, ZA, 68, 317

Girardi L., Eggenberger P., \& Miglio A., 2011, MNRAS, 412, L103

Goldreich P., \& Schubert G., 1967, ApJ, 150, 571

Goudfrooij P., Puzia T. H., Kozhurina-Platais V., \& Chandar R., 2009, AJ, 137, 4988

Goudfrooij P., Puzia T. H., Kozhurina-Platais V., \& Chandar R., 2011, ApJ, 737, 3

Iglesias C., \& Rogers F. J., 1996, ApJ, 464, 943

Kawaler S. D., 1988, ApJ, 333, 236

Kippenhahn R., \& Thomas H. C, 1970, in Stellar Rotation, ed. A. Slettebak (New York: Gordon and Breach), 20

Kosovichev A. G. et al., 1997, Sol. Phys., 170, 43

Mackey A. D., \& Broby Nielsen P., 2007, MNRAS, 379, 151

Mackey A. D., Broby Nielsen P., Ferguson A. M. N., \& Richardson J. C., 2008, ApJ, 681, L17

Maeder A., \& Meynet G., 2000, ARA\&A, 38, 143

Meynet G., \& Maeder A., 1997, A\&A, 321, 465

Milone A. P., Bedin L. R., Piotto G., \& Anderson J., 2009, A\&A, 497, 755

Pinsonneault M. H., Kawaler S. D., Sofia S., \& Demarqure P., 1989, ApJ, 338, 424

Platais I. et al., 2012, ApJ, 751, L8

Rogers F. J., \& Nayfonov A., 2002, ApJ, 576,1064

Royer F., Zorec J., \& Gómez A. E., 2007, A\&A, 463, 671

Rubele S., Girardi L., Kozhurina-Platais V., Goudfrooij P., \& Kerber L., 2011, MNRAS, 414, 2204

Rubele S., Kerber L., \& Girardi L., 2010, MNRAS, 403, 1156 
Talon S., \& Charbonnel C., 2005, A\&A, 440, 981

Thoul A. A., Bahcall J. N., \& Loeb A., 1994, ApJ, 421, 828

Von Zeipel H., 1924, MNRAS, 84, 665

Yang W. M., \& Bi S. L., 2006, A\&A, 449, 1161

Yang W. M., \& Bi S. L., 2007, ApJ, 658, L67

Yang W. M., \& Bi S. L., 2008, Chjaa, 8, 677

Yang W. M., Li Z. M., Meng X. C., \& Bi S. L., 2011a, MNRAS, 414, 1769

Yang W. M., Meng X. C., Bi S. L., Tian Z., Li T., \& Liu K., 2011b, ApJ, 731, L37

Yang W. M., Meng X. C., Bi S. L., Tian Z., Liu K., Li T., \& Li Z., 2012, MNRAS, 422, 1552

Yoon S. C., Langer N., \& Norman C., 2006, A\&A, 460, 199

Zahn J.-P., 1992, A\&A, 265, 115

Zahn J.-P., Talon S., \& Matias J., 1997, A\&A, 322, 320

Zorec J., \& Royer F., 2012, A\&A, 537, A120

This paper was prepared with the RAA LATEX macro v1.2. 